Review Article

\title{
Role of The Two Highly Conserved Features of Initiator tRNAs in Initiation of Protein Synthesis in Eubacteria
}

\author{
RIYAZ AHMAD SHAH ${ }^{1}$, SUNIL SHETTY ${ }^{1}$ and UMESH VARSHNEY ${ }^{1,2, *}$ \\ ${ }^{1}$ Department of Microbiology and Cell Biology, Indian Institute of Science, Bangalore 560 012, India \\ ${ }^{2}$ Jawaharlal Nehru Centre for Advanced Scientific Research, Jakkur, Bangalore 560 064, India
}

(Received on 12 December 2017; Revised on 01 January 2018; Accepted on 25 January 2018)

\begin{abstract}
Regulation of translation represents a crucial step in gene expression. In this mini-review, we provide a brief outline of protein synthesis in bacteria, and discuss the participation of a special tRNA, the initiator tRNA (i-tRNA), in the different stages of the initiation step. The conserved features of the bacterial i-tRNA are located in its acceptor and anticodon stems. A Watson-Crick mismatch pair $(1 \times 72)$ at the top of the acceptor stem along with the 2:71 and 3:70 pairs, is important in formylation of the amino acid attached to the i-tRNA. The property of formylation facilitates i-tRNA binding to the $30 \mathrm{~S}$ ribosome. The second feature of the presence of the three consecutive G:C pairs $(29: 41 ; 30: 40$ and 31:39 positions) in the anticodon stem, highly conserved in the i-tRNAs in all domains of life, plays a crucial role in the transition of i-tRNA through the different stages in formation of the $70 \mathrm{~S}$ elongation competent complex from the $30 \mathrm{~S}$ pre-initiation complex.
\end{abstract}

Keywords: Initiator tRNAs; Protein Synthesis

\section{Introduction}

Translation of mRNA into protein, a major hub for regulation of gene expression, comprises initiation, elongation, termination and recycling steps and is catalyzed by large ribonucleoprotein complexes called ribosomes. Bacterial ribosomes comprise a large $(\sim 1.5$ MDa; 50S) and a small ( 0.85 MDa; 30S) subunits made of ribosomal proteins (r-proteins) and RNA (rRNA) which together constitute 70S ribosome with E-, P- and A- sites for binding transfer RNAs (tRNAs). The tRNAs facilitate the process of translation by serving as adaptor molecules that bring amino acids (aa) to the ribosome in response to the array of the triplet nucleotides (codon) in the messenger RNA (mRNA). Precise reading of the codons in mRNA that begins with the identification and recognition of the first codon, the start or the initiation codon (during the step of initiation) is crucial for production of a meaningful protein (Laursen et al., 2005). Thus, cells possess two kinds of tRNAs, an initiator tRNA (itRNA, a special tRNA which binds directly in the P- site to read the start codon) and the elongator tRNAs (that bind in the A-site to decode the subsequent codons). Selection of the correct aa-tRNA in the Asite of the ribosome is a key process that ensures fidelity of translation. Ribosomes catalyze repetitive reactions of transferring the aa or the peptide from the P-site tRNA to the aa on the A-site tRNA by peptide bond formation during elongation (Fischer $e t$ al., 2010). The A-site tRNA (now attached with a peptide) is moved to the $\mathrm{P}$-site and, the deacylated $\mathrm{P}$ site tRNA is simultaneously moved to the E (exit)site together with the concerted movement of mRNA (translocation), exposing the next codon in A-site to bind another aa-tRNA. The peptide bond formation results in location of the tRNAs in the hybrid states, the P-site tRNA originally in the P/P state (P-site of 30S and P-site of 50S) transitions into the P/E state (P-site of 30S and E-site of 50S), and the A-site tRNA bound in the A/A state, into the A/P state together with the anticlockwise movement (ratcheting) of the $30 \mathrm{~S}$ subunit, resulting in a rotated state of the ribosome. The process of translocation is crucial to convert the 
rotated ribosomes back to the nonrotated state and to transfer the $\mathrm{P} / \mathrm{E}$ and $\mathrm{A} / \mathrm{P}$ hybrid state tRNAs into the $\mathrm{E} / \mathrm{E}$ and $\mathrm{P} / \mathrm{P}$ states to expose a new codon in the Asite (Dunkle et al., 2011; Fischer et al., 2010). Arrival of a special codon (termination codon) at the end of mRNA reading frame in the A-site, prompts the release of the synthesized polypeptide, and dissociation of the ribosomal subunits from the mRNA to begin a new round of protein synthesis. Numerous protein factors assist the energy consuming process of protein synthesis. Initiation factors (IF1, IF2 and IF3) play crucial roles during initiation; elongation factors EFTu and EFG help recruitment and movement (translocation) of tRNAs, respectively; release factors (RF1, RF2 and RF3) facilitate release of the newly synthesized polypeptide; and the ribosome recycling factor (RRF) together with EFG and IF3 separates the ribosomal subunits from the mRNA (Schmeing and Ramakrishnan, 2009).

Translation initiation is a rate limiting step in protein synthesis and subject to various regulatory mechanisms in all kingdoms of life (Gold, 1988). The canonical pathway of initiation of protein synthesis in bacteria makes use of the three highly conserved and essential initiation factors (IF1, IF2 and IF3); and, binding of mRNA to the 30S subunit is facilitated by the interaction of a purine rich sequence (Shine Dalgarno sequence, SD) in mRNA (occurring upstream of the start codon) with a sequence towards the end of 16S rRNA in the 30S subunit. The i-tRNA binds in the P-site and its anticodon pairs with the start codon in the mRNA. The initiation factors, particularly IF3 ensures fidelity of i-tRNA binding in the P-site and recognition of the start codon. The complex of 30S ribosome with i-tRNA, mRNA, and the initiation factors is designated as $30 \mathrm{~S}$ pre-initiation complex (30S PIC), which is then joined by the 50S subunit to form a $70 \mathrm{~S}$ complex, that is finally converted into an elongation competant 70S complex (70S EC) upon the release of the initiation factors. The 70S EC possesses the i-tRNA bound in the P-sites of both the $30 \mathrm{~S}$ and $50 \mathrm{~S}$ subunits (P/P state) and has the A-site available to bind to the aa-tRNA specified by the mRNA codon in the A-site (Figs. 1, 2).

Recent single molecule studies show that there can be multiple pathways to reach the formation of 30S PIC (Tsai et al., 2012). In cryo-electron microscopic structures of 30S PIC, it was observed that $\mathrm{i}$-tRNA is not found in the P/P state as in the 70S $\mathrm{EC}$, instead it is slightly tilted towards E-site of the ribosomes (P/I state) (Julian et al., 2011). Further, loading of the large ribosomal subunit (50S) might happen in this stage only. Single molecule studies have also suggested that hydrolysis of IF 2 bound GTP upon 50S subunit binding causes rotational movement (opposite to the usual ratcheting occuring during the elongation step) of the two subunits in such a way that $\mathrm{i}$-tRNA is now in $\mathrm{P} / \mathrm{P}$ state. This in turn, triggers

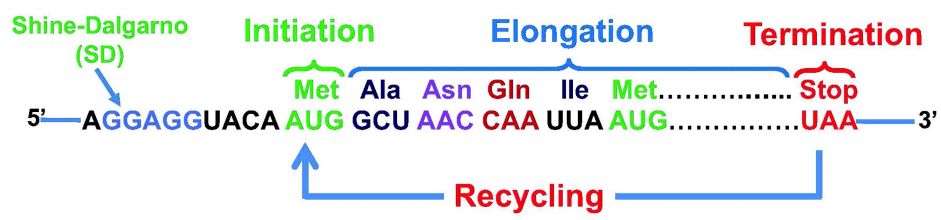

Fig. 1: Schematic of translation of mRNA

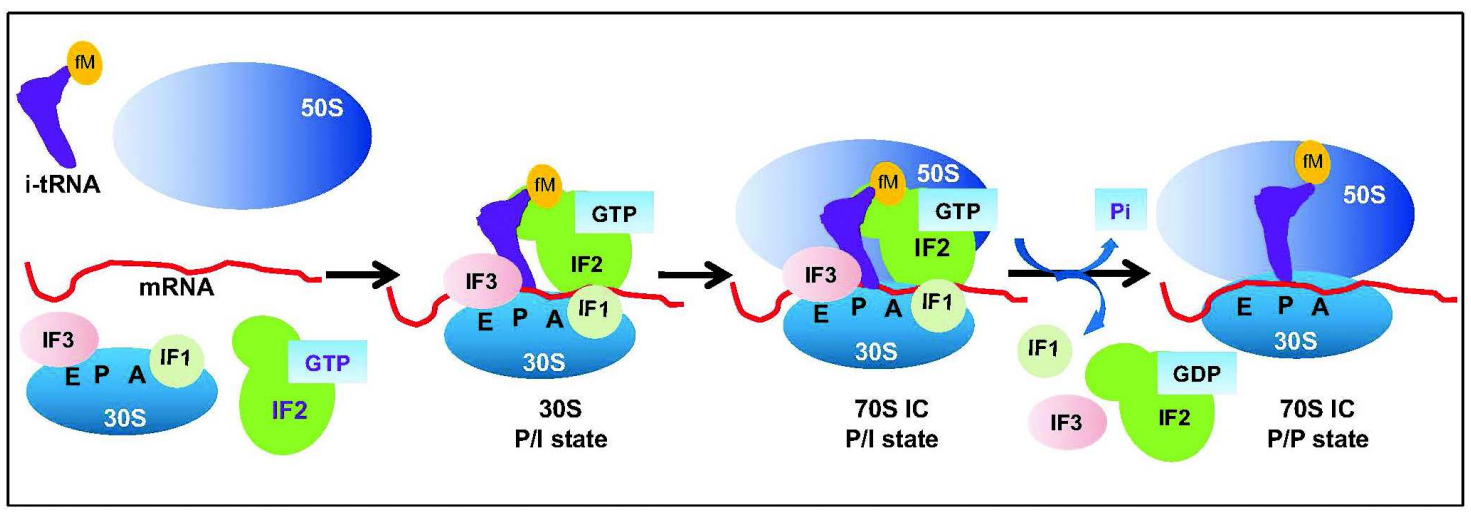

Fig. 2: Various stages in translation initiation in eubacteria 
the release of IF1, IF2, as well as IF3 to produce 70S EC (Marshall et al., 2009; Tsai et al., 2012). Thus, initiation process possesses distinct stages defined by many conformational changes.

Our research activities have focused on the process of selection of the i-tRNA in the P-site and its participation through the multiple stages in initiation. Thus, i-tRNA provides us with an excellent probe to understand the mechanism of initiation. Here, we discuss how the salient features of i-tRNA contribute to the various reactions in the process of initiation, primarily based on our own findings.

\section{Initiator tRNA and Its Unique Features}

E. coli chromosome possesses four copies of i-tRNA genes. Three of them (met $Z W V$ ) are located at 63.5 $\mathrm{min}$, whereas the fourth one (met $Y$ ) is located some distance away at $71.5 \mathrm{~min}$. Selection of i-tRNA (Fig. 2) in the P-site of the ribosome is primarily assisted by its unique features and the various IFs (RajBhandary, 1994). The two important features of i-tRNAs are, (a) the presence of a Watson-Crick mismatch pair at the $1 \times 72$ position in the acceptor stem, and, (b) the presence of three consecutive $\mathrm{G}: \mathrm{C}$ base pairs (GC/GC/GC, 3GC pairs) at 29:41, 30:40, 31:39 positions in the anticodon stem of i-tRNA (Fig. 3; (Mandal et al., 1996; Marck and Grosjean, 2002)). IF2 has much higher affinity for the formyl-aa-tRNA and facilitates binding of $\mathrm{i}$-tRNA to the P-site (Mangroo and Raj Bhandary, 1995; Wu and Raj Bhandary, 1997). IF3, an anti-association factor in E. coli, has a major role in selection of i-tRNAs (Antoun et al., 2006a; Antoun et al., 2006b; Karimi et al., 1999). IF1 has also been proposed to enhance initiation by favoring binding of IF2 (Antoun et al., 2006a; Antoun et al., 2006b). Thus, all the three initiation factors make the ribosomal $\mathrm{P}$-site selective for i-tRNA binding. In addition, the tails of the r-proteins S9 and S13 and the highly conserved 16S rRNA nucleotides, G1338 and A1339, in the P-site have been shown to be crucial in the selection of i-tRNA in the P-site (Arora et al., 2013a; Arora et al., 2013b; Lancaster and Noller, 2005; Selmer et al., 2006).

\section{Mismatch at the $1 \times 72$ Position of the i-tRNA and the Role of Formylation}

The mismatch at the top of the acceptor stem (position $1: 72$ ) is conserved in i-tRNAs from eubacteria
(C1xA72 in E. coli) and is not found in the elongator tRNAs. This mismatched pair contributes to several of the required properties of the i-tRNAs and provides a crucial determinant for formylation of the aa attached to i-tRNA, by Fmt (10-Formyl tetrahydrofolate methionine transferase or formylase) (Guillon et al., 1992b; Lee et al., 1992; Lee et al., 1991). The formylation reaction increases the affinity of i-tRNA to IF2 (Sundari et al., 1976). An efficient rate of formylation of the aminoacylated i-tRNA also avoids its partitioning with EFTu, and its participation in elongation (Nissen et al., 1995; Seong and RajBhandary, 1987b). Single molecule studies suggested that formylation enhances the formation of $30 \mathrm{~S}$ initiation complex and the stability of the 70S complex (Ling and Ermolenko, 2015; Masuda et al., 2012). Although the lack of Fmt causes severe growth retardation of bacteria, it is not absolutely essential in E. coli under the laboratory culture conditions (Guillon et al., 1992a). Interestingly, the growth defect in bacteria occuring due to the lack of Fmt is rescued by the amplification of gene copy numbers in the $\mathrm{i}$ tRNA loci (Nilsson et al., 2006). Likewise, overexpression of i-tRNA from plasmid borne i-tRNA genes also rescues for the deficiency of formylation (Shetty et al., 2017). These observations suggest that the formylation of aminoacylated i-tRNA primarily supports at some of the very early steps, such as its initial recruitment to the $30 \mathrm{~S}$ ribosome. The high copy number of i-tRNA genes in most organisms ( 4 in $E$. coli) also contributes to prevention of elongator tRNA binding to the P-site (Kapoor et al., 2011). Thus, formylation is crucial not only for the higher efficiency of initiation but also for the high fidelity of initiation. In case of eukaryotes, there is no formylation of aa attached to the i-tRNA. However, the A1:U72 base pair enhances its binding to eIF2-GTP (Farruggio et al., 1996; Kapp and Lorsch, 2004) as well as the binding of the ternary complex (aminoacylated itRNA, eIF2-GTP) to 40S pre-initiation complex (Kapp et al., 2006). It is also needed for survival of yeast cells (Astrom et al., 1993; von PawelRammingen et al., 1992).

\section{Role of the 3GC Base Pairs}

Unlike formylation, which is conserved only in eubacteria, the presence of $3 \mathrm{GC}$ base pairs in the anticodon stems of $\mathrm{i}$-tRNAs is highly conserved in all domains of life (barring some exceptions), suggesting 
a critical role for this feature in the process of initiation. In fact, using both in vivo and in vitro experiments, it has been shown that the lack of the $3 \mathrm{GC}$ pairs renders the i-tRNA mutants inefficient in translation initiation or in their stable binding to ribosomes (Mandal et al., 1996; Seong and RajBhandary, 1987a; Varshney et $a l ., 1991)$. Although the precise role of the 3GC pairs has remained unclear, in the crystal structures of $70 \mathrm{~S}$ ribosomes containing mRNA and i-tRNA in the Psite, the two universally conserved residues G1338 and A1339 of 16S rRNA (G1575 and A1576 of 18S rRNA in yeast) are poised to make A-minor interactions with the minor groove sides of the first and the second G:C base pairs (Korostelev et al., 2006; Selmer et al., 2006). In vitro studies showed that these interactions stabilize the binding of i-tRNA in the ribosomal P-site (Lancaster and Noller, 2005; Qin et al., 2007).

\section{Naturally Occurring Exceptions to the '3GC Rule' and the Minimalist '3GC Rule'}

We observed that at least some of the mycoplasmal and rhizobial species possess variations in the $3 \mathrm{GC}$ pairs of i-tRNAs (Fig. 3). These natural variants possess either AU in place of the first GC $\left(1^{\text {st }} \mathrm{AU}\right.$, or

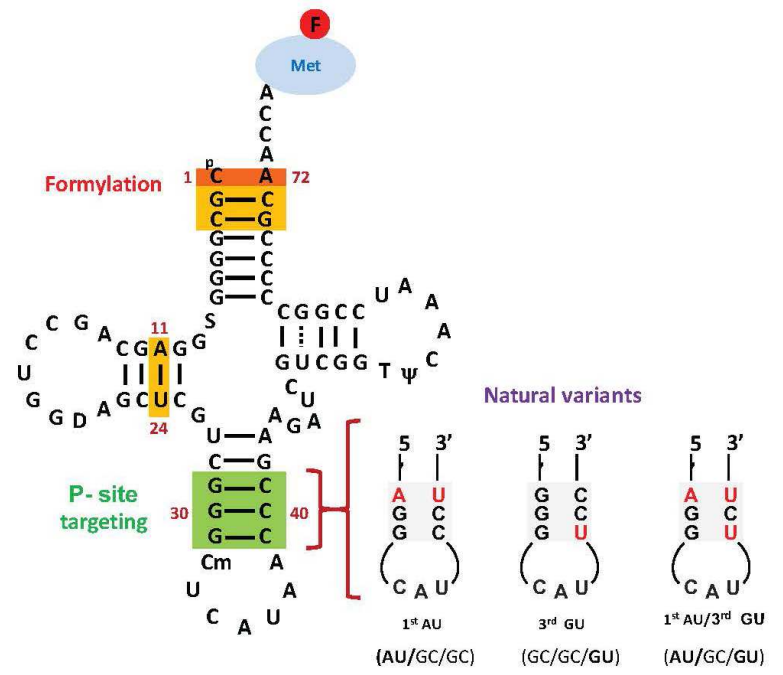

Fig. 3: Sequence and clover leaf structure of initiator tRNA and its naturally occurring anti-codon stem variants. The 3GC base pairs are highlighted in green, the formylation features are highlighted in yellow/ orange. The presence of a mismatch at the $1 \times 72$ position in the acceptor stem is shown highlighted in orange. Mycoplasmal and rhizobial natural variants of initiator tRNAs are shown highlighted in grey and labelled according to the variation present
AU/GC/GC), GU in place of the third GC ( $3^{\text {rd }} \mathrm{GU}$, GC/GC/GU) or both (AU/GC/GU) (Samhita et al., 2012); (Fig. 3). These observations raise a question on the essentiality of the occurrence of the $3 \mathrm{GC}$ pairs. Thus, to understand the importance of the $3 \mathrm{GC}$ pairs and to investigate if these (mycoplasmal and rhizobial) unconventional i-tRNAs could function in E. coli, we used the approach of deleting all the four i-tRNA genes from $E$. coli genome in the presence of plasmid borne copies of the unconventional i-tRNA genes. As the presence of functional i-tRNA is essential for $E$. coli growth, deletion of all the i-tRNA genes (metZWV and metY) would materialize only if the variant $\mathrm{i}$-tRNAs (plasmid borne) were functional in E. coli. The i-tRNAs with naturally occurring variants of the $3 \mathrm{GC}$ pairs sustained $E$. coli for the deletion of $m e t Z W V$ and met $Y$. However, those that contained either the sequence corresponding to that of the elongator tRNA ${ }^{\text {Met }}$ (i.e. UA/CG/AU) or GC/CG/ AU or GC/CG/GC failed to do so (Samhita et al., 2012). Interestingly, even though the naturally occurring variants of i-tRNAs which sustained $E$. coli growth did not possess $3 \mathrm{GC}$ pairs, they still possessed a sequence with 3(Purine:Pyrimidine) pairs. This observation raised the possibility of requirement of three Purine:Pyrimidine base pairs rather than the 3GC pairs as the essential feature for the i-tRNA function. Extensive mutagenesis of individual GC pairs into other base pairs such as AU and CG pairs suggested that among the three base pair positions, first and third pairs could be changed to AU or CG pairs, thus requirement of three Purine:Pyrimidine pairs was also ruled out. However, the mutants containing either $\mathrm{AU}$ or CG pair in place of the middle GC pair did not sustain E. coli. This implied that middle GC pair is essential for i-tRNA function (Fig. 4; (Shetty et al., 2017). The mutants containing AU, CG or GU in the $1^{\text {st }}$ or the $3^{\text {rd }}$ base pair positions in different combinations sustained $E$. coli in the context of middle GC pair.

The i-tRNA mutant with all its $3 \mathrm{GC}$ pairs replaced with AU pairs (AU/AU/AU) did not sustain E. coli for its growth. However, conversion of middle AU to GU (by mutating A30 back to G30) in the AU/ GU/AU mutant allowed it to sustain $E$. coli growth revealing that the role of G30 is the most crucial. Although it should also be said that the middle GU pair did not support $E$. coli growth when it occurred in the context of the flanking pairs as CG (i.e. CG/ 


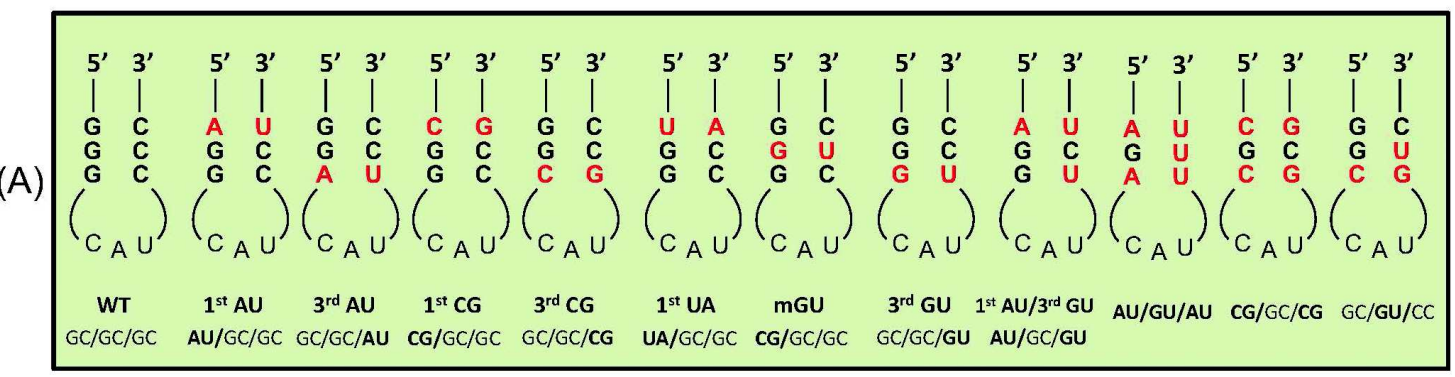

(B)

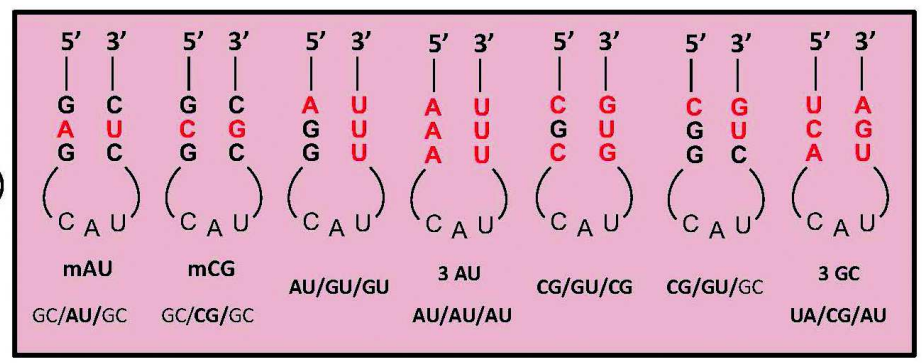

Fig. 4: Anticodon stem loop of wild type and mutant initiator tRNAs. Wild type initiator tRNA anticodon stem containing full complement of 3GC base pairs is shown as WT, mutations in the 3GC base pairs are shown in red and labelled according to the mutation. The mutants of 3GC base pairs that sustain $E$. coli lacking all the four copies of genomically encoded i-tRNA are shown in the green box, while mutants which could not sustain the cell are shown in the red box

GU/CG). Moreover, while the GC/GU/CG mutant supported E. coli growth, the CG/GU/GC did not, suggesting that the middle GU pair can function only in the context of a Purine:Pyrimidine pair at the $1^{\text {st }} \mathrm{GC}$ position, i.e. either GC or AU pair. These observations suggest that presence of not just G30 but also the right structural context for it are crucial. Structural and molecular dynamic analyses might facilitate further understanding on the presentation of the G30 residue in the correct structural context. It may also be noted that the $U$ residues in positions 38,39 and 40 could be pseudouridylated by TruA (Hur and Stroud, 2007) and it would be of interest to investigate what role(s) such a modification might have.

Another question that arises from our mutational analyses is that if the middle GC or even G30 in an appropriate context are adequate to sustain E. coli, why the 3GC pairs have been so highly conserved in all domains of life. Importantly, when we analysed relative growth of $E$. coli strains sustained on different i-tRNA mutants, we noted differences in growth especially at cold temperature as well as on minimal media, suggesting that the presence of a full complement of the three GC base pairs confers a fitness advantage for growth under the conditions of stress (Shetty et al., 2017). Further, many elongator tRNAs do possess the middle GC pair (together with the $1^{\text {st }}$ or the $3^{\text {rd }} \mathrm{GC}$ pair) in their anticodon stems. Thus, it would be of interest to see how such elongator tRNAs would compete with the i-tRNA, perhaps in the conditions of stress.

Importance of the 3GC pairs has also been investigated for yeast i-tRNA by similar approach of sustainability using plasmid encoded mutant i-tRNAs (Dong et al., 2014; von Pawel-Rammingen et al., 1992). Yeast provides an interesting system since in this organism both the initiator and elongator species of tRNA ${ }^{\text {Met }}$ possess the middle GC pair. Mutations in the $1^{\text {st }}$ and the $3^{\text {rd }} \mathrm{GC}$ pairs revealed that changing GC pairs into even U:U pairs did not hamper the ability of the i-tRNA mutant to sustain yeast (von PawelRammingen et al., 1992). However, the in vitro reconstituted experiments suggested that the GC pairs are important for formation of 40S IC (Kapp et al., 2006). Further, it was shown that the $3 \mathrm{GC}$ pairs are essential in yeast (Dong et al., 2014). The study showed that neither of the 3 positions tolerated Purine:Purine pairs. Also, neither the $1^{\text {st }}$ nor the $2^{\text {nd }}$ GC pairs tolerated Pyrimidine:Pyrimidne pairs. However, the $3^{\text {rd }}$ GC could be changed to Pyrimidine:Pyrimidine pairs to still retain the biological function of the mutant. Interestingly, in contrast to $E$. coli, in yeast i-tRNA, substitution of the middle GC pair with either AU or CG pairs still retained its 
biological function to sustain yeast. In eukaryotes, scanning mode of initiation occurs where the 40S IC containing i-tRNA scans the 5' UTR and recognizes the start codon (Hinnebusch and Lorsch, 2012). During scanning, the 40S complex is in open conformation, while upon recognition of the start codon it attains closed conformation. As the process of initiation in yeast is mechanistically different, the exact requirement of the GC pairs might also be different. Extensive mutagenesis of the $3 \mathrm{GC}$ pairs in i-tRNA would be necessary to infer their precise role in yeast.

\section{Role of the 3GC Pairs in Formation of $70 S$ Complex}

In vitro studies suggested that the lack of the $3 \mathrm{GC}$ pairs affects the stability of 30S IC (Hartz et al., 1989). The in vivo reporter assays suggested that the $3 \mathrm{GC}$ pairs are crucial for translation initiation (Mandal et $a l .$, 1996). Further, the in vivo analysis of abundance of i-tRNA across the polysome profiles showed that most of the wild type or the mutant i-tRNAs that support E. coli growth accumulate in the $70 \mathrm{~S}$ ribosome population (with a much less of the i-tRNA fraction occurring in the $30 \mathrm{~S}$ population). However, for an i-tRNA mutant unable to support $E$. coli growth (e. g. the UA/CG/AU mutant), most of the tRNA was found in the $30 \mathrm{~S}$ population and very little in the 70S (Shetty et al., 2014). These observations suggested that the $3 \mathrm{GC}$ pairs are crucial for transition of the i-tRNA from the $30 \mathrm{~S}$ to $70 \mathrm{~S}$ complexes. Also, unlike the rescue of the growth defect of $E$. coli due to the lack of the formylation of i-tRNA by increasing the amount of i-tRNA in the cells, overexpression of 3GC mutant i-tRNAs did not mitigate the growth defect of the cells harboring i-tRNAs defective in initiation due to mutation(s) in the 3GC sequence (Shetty et al., 2017). These observations suggested that while the major role of formylation is in the initial binding of i-tRNA to the ribosome, that of the 3GC pairs is in the later stages of initiation. Thus, we proposed sequential roles of these two unique features, formylation being crucial in the recruitment of i-tRNA to the $\mathrm{P}$-site, and the $3 \mathrm{GC}$ pairs governing the later steps of transition into the 70S EC (Fig. 5). Further, a genetic suppressor analysis suggested that the presence of an extended SD context in them RNA facilitated the availability of the 3GC mutant i-tRNA into the 70S complex (Shetty et al., 2014).
Analyses with many other i-tRNAs mutated in their $3 \mathrm{GC}$ pairs suggested that while the $1^{\text {st }}$ and the $3^{\text {rd }} \mathrm{GC}$ pair position mutants enter the $70 \mathrm{~S}$ complex efficiently, the mid AU mutants showed a major defect in their transition to 70S (Shetty et al., 2017). Interestingly, while the 3AU (AU/AU/AU) mutant failed to enter the 70S complex efficiently, reintroduction of $\mathrm{G}$ at position 30 (AU/GU/AU) allowed the i-tRNA to enter $70 \mathrm{~S}$ complex suggesting an essential role of the G30 in the mid GC pair in the formation of the 70S complex. Surprisingly, while the midCG mutant is capable of entering the 70S complex efficiently, it fails to sustain $E$. coli for its growth. This observation prompted us to investigate for the additional roles of the $3 \mathrm{GC}$ pairs.

\section{The 3GC Base Pairs and IF3 Release}

Among the three initiation factors, IF3 plays a crucial role in the fidelity of initiation. Mutations in IF3 have been shown to affect 3GC pair discrimination as well as start codon selection (Ayyub et al., 2017; O'Connor et al., 2001; Petrelli et al., 2003; Petrelli et al., 2001). In vitro studies had suggested that IF3 destabilises elongator tRNA containing 70S IC (Antoun et al., 2006a; Antoun et al., 2006b). Further, it has been shown that IF3 discriminates the $3 \mathrm{GC}$ pairs by causing structural rearrangements in the $16 \mathrm{~S}$ rRNA loop containning G1338, A1339 (Lancaster and Noller, 2005). It has also been proposed that in the presence of IF3, G1338 and A1339 residues allow only tRNA with the $3 \mathrm{GC}$ pairs to form initiation complex. Interestingly, when we analysed the $70 \mathrm{~S}$ complexes from E. coli (supported by the chromosomal copy of i-tRNA gene) harboring midCG mutants, they retained more IF3 in 70S complex compared to the 70S complexes formed with the wild type or the i-tRNA mutants that support E. coli for its growth (Shetty et al., 2017). Thus, although the mid CG i-tRNA mutant is able to transit into the 70S complex, it is defective in releasing IF3 from 70 S, and thus in sustaining $E$. coli for its growth.

\section{Conclusions and Future Perspectives}

The two most distinctive elements of eubacterial itRNAs are located in their acceptor and the anticodon stems (RajBhandary, 1994; Varshney and Raj Bhandary, 1992). The mismatched base pair at the top of the acceptor stem $(1 \times 72)$ along with the $2: 71$ and 3:70 pairs in the acceptor stem are responsible 


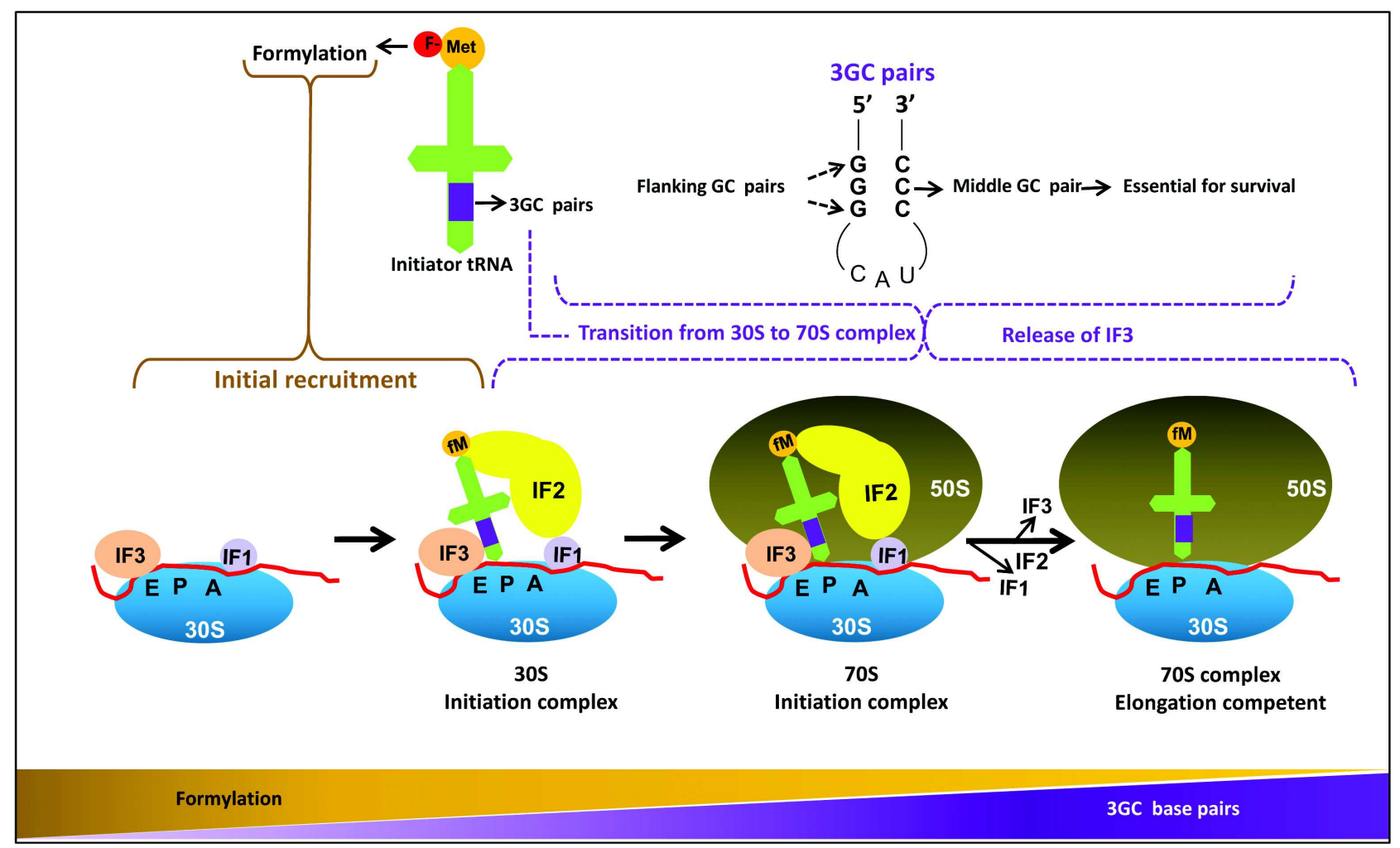

Fig. 5: Model showing the major roles of formylation and 3GC base pairs of initiator tRNA at different stages of initiation

for the recognition of an i-tRNA by Fmt for the formylation of the attached aa. The function of formylation of i-tRNA is primarily to facilitate its binding to IF2 and to the $30 \mathrm{~S}$ ribosome. The second feature of the presence of the three consecutive G:C base pairs (3GC pairs) in the anticodon stem is highly conserved in all domains of life. In our earlier studies, we showed that incorporation of these two distinctive features of i-tRNAs into an elongator tRNA conferred upon it the initiation activity (Varshney et al., 1993). In our recent studies, we have shown that the feature of the $3 \mathrm{GC}$ pairs, facilitates transition of the i-tRNA from the $30 \mathrm{~S}$ ribosome to the $70 \mathrm{~S}$ ribosome. In addition, we observed that the $3 \mathrm{GC}$ base pairs also facilitate release of IF3 from the 70S PIC (Fig. 5; Shetty et al., 2017). More recently, we observed that the pioneering round of initiation is also responsible to prompt ultimate steps of the ribosome maturation by signaling RNases to trim the terminal extensions of immature 16S rRNA (Shetty and Varshney, 2016). The cellular level of i-tRNAs are subject to regulation under stress (ArgR, ppGpp, VapC) (Krin et al., 2003; Nagase et al., 1988; Winther and Gerdes, 2011). Does the deficiency of i-tRNA in cells lead to generation of ribosomal heterogeneity due to deficiency in the processing of the 16S rRNA at the 5'-, and the 3'ends? Do the ribosomes with heterogenous ends influence translation of cellular mRNAs (e. g. by impacting SD anti-SD interaction)? Interestingly, it has also been shown that a slight deficiency of i-tRNA in E. coli confers fitness advantage for growth under nutrient limiting conditions (Samhita et al., 2014). Investigation of how SD and anti-SD interactions impact the requirement of the $3 \mathrm{GC}$ pairs in i-tRNA, especially when such interactions are established within the rRNA of the small ribosomal subunit in the unprocessed regions in the intermediates (e. g. 17S rRNA) that produce mature 16S rRNA (Shetty et al., 2014) would be of interest. Furthermore, the study of mechanistic aspects of how $3 \mathrm{GC}$ pairs are involved in IF3 release would be of much importance. 


\section{References}

Antoun A, Pavlov M Y, Lovmar M and Ehrenberg M (2006a) How initiation factors maximize the accuracy of tRNA selection in initiation of bacterial protein synthesis Molecular cell 23 183-193

Antoun A, Pavlov M Y, Lovmar M and Ehrenberg M (2006b) How initiation factors tune the rate of initiation of protein synthesis in bacteria The EMBO journal 25 2539-2550

Arora S, Bhamidimarri S P, Bhattacharyya M, Govindan A, Weber M H, et al. (2013a) Distinctive contributions of the ribosomal P-site elements m2G966, m5C967 and the Cterminal tail of the $\mathrm{S} 9$ protein in the fidelity of initiation of translation in Escherichia coli Nucleic Acids Res $414963-$ 4975

Arora S, Bhamidimarri S P, Weber M H and Varshney U (2013b) Role of the ribosomal P-site elements of $\mathrm{m}(2) \mathrm{G} 966$, $\mathrm{m}(5) \mathrm{C} 967$, and the S9 C-terminal tail in maintenance of the reading frame during translational elongation in Escherichia coli Journal of bacteriology 195 3524-3530

Astrom S U, von Pawel-Rammingen U and Bystrom A S (1993) The yeast initiator tRNAMet can act as an elongator tRNA(Met) in vivo Journal of molecular biology 233 4358

Ayyub S A, Dobriyal D and Varshney U (2017) Contributions of the $\mathrm{N}$ - and C-Terminal Domains of Initiation Factor 3 to Its Functions in the Fidelity of Initiation and Antiassociation of the Ribosomal Subunits Journal of bacteriology 199

Dong J, Munoz A, Kolitz S E, Saini A K, Chiu W L, et al. (2014) Conserved residues in yeast initiator tRNA calibrate initiation accuracy by regulating preinitiation complex stability at the start codon Genes \& development 28502 520

Dunkle J A, Wang L, Feldman M B, Pulk A, Chen V B, et al. (2011) Structures of the bacterial ribosome in classical and hybrid states of tRNA binding Science 332 981-984

Farruggio D, Chaudhuri J, Maitra U and RajBhandary U L (1996) The A 1 x U72 base pair conserved in eukaryotic initiator tRNAs is important specifically for binding to the eukaryotic translation initiation factor eIF2 Molecular and cellular biology 16 4248-4256

Fischer N, Konevega A L, Wintermeyer W, Rodnina M V and Stark H (2010) Ribosome dynamics and tRNA movement by time-resolved electron cryomicroscopy Nature 466 329-333

Gold L (1988) Posttranscriptional regulatory mechanisms in Escherichia coli Annual review of biochemistry 57 199233
Guillon J M, Mechulam Y, Schmitter J M, Blanquet S and Fayat G (1992a) Disruption of the gene for Met-tRNA(fMet) formyltransferase severely impairs growth of Escherichia coli Journal of bacteriology 174 4294-4301

Guillon J M, Meinnel T, Mechulam Y, Lazennec C, Blanquet S, et al. (1992b) Nucleotides of tRNA governing the specificity of Escherichia coli methionyl-tRNA(fMet) formyltransferase Journal of molecular biology 224359 367

Hartz D, McPheeters D S and Gold L (1989) Selection of the initiator tRNA by Escherichia coli initiation factors Genes \& development 3 1899-1912

Hinnebusch A G and Lorsch J R (2012) The mechanism of eukaryotic translation initiation: new insights and challenges Cold Spring Harbor perspectives in biology 4

Hur S and Stroud R M (2007) How U38, 39, and 40 of many tRNAs become the targets for pseudouridylation by TruA Molecular cell 26 189-203

Julian P, Milon P, Agirrezabala X, Lasso G, Gil D, et al. (2011) The Cryo-EM structure of a complete $30 \mathrm{~S}$ translation initiation complex from Escherichia coli PLoS biology 9 e1001095

Kapoor S, Das G and Varshney U (2011) Crucial contribution of the multiple copies of the initiator tRNA genes in the fidelity of tRNA(fMet) selection on the ribosomal P-site in Escherichia coli Nucleic Acids Res 39 202-212

Kapp L D, Kolitz S E and Lorsch J R (2006) Yeast initiator tRNA identity elements cooperate to influence multiple steps of translation initiation Rna 12 751-764

Kapp L D and Lorsch J R (2004) GTP-dependent recognition of the methionine moiety on initiator tRNA by translation factor eIF2 Journal of molecular biology 335 923-936

Karimi R, Pavlov M Y, Buckingham R H and Ehrenberg M (1999) Novel roles for classical factors at the interface between translation termination and initiation Molecular cell 3 601609

Korostelev A, Trakhanov S, Laurberg M and Noller H F (2006) Crystal structure of a 70S ribosome-tRNA complex reveals functional interactions and rearrangements Cell 1261065 1077

Krin E, Laurent-Winter C, Bertin P N, Danchin A and Kolb A (2003) Transcription regulation coupling of the divergent $\operatorname{argG}$ and metY promoters in Escherichia coli K-12 Journal of bacteriology 185 3139-3146

Lancaster L and Noller H F (2005) Involvement of 16S rRNA nucleotides G1338 and A1339 in discrimination of initiator tRNA Mol Cell 20 623-632 
Laursen B S, Sorensen H P, Mortensen K K and Sperling-Petersen $\mathrm{H} \mathrm{U}$ (2005) Initiation of protein synthesis in bacteria Microbiol Mol Biol Rev 69 101-123

Lee C P, Dyson M R, Mandal N, Varshney U, Bahramian B, et al. (1992) Striking effects of coupling mutations in the acceptor stem on recognition of tRNAs by Escherichia coli Met-tRNA synthetase and Met-tRNA transformylase Proceedings of the National Academy of Sciences of the United States of America $\mathbf{8 9} 9262-9266$

Lee C P, Seong B L and RajBhandary U L (1991) Structural and sequence elements important for recognition of Escherichia coli formylmethionine tRNA by methionyl-tRNA transformylase are clustered in the acceptor stem The Journal of biological chemistry 266 18012-18017

Ling C and Ermolenko D N (2015) Initiation factor 2 stabilizes the ribosome in a semirotated conformation Proceedings of the National Academy of Sciences of the United States of America 112 15874-15879

Mandal N, Mangroo D, Dalluge J J, McCloskey J A and Rajbhandary U L (1996) Role of the three consecutive G:C base pairs conserved in the anticodon stem of initiator tRNAs in initiation of protein synthesis in Escherichia coli Rna 2 473-482

Mangroo D and RajBhandary U L (1995) Mutants of Escherichia coli initiator tRNA defective in initiation. Effects of overproduction of methionyl-tRNA transformylase and the initiation factors IF2 and IF3 J Biol Chem 270 1220312209

Marck C and Grosjean H (2002) tRNomics: analysis of tRNA genes from 50 genomes of Eukarya, Archaea, and Bacteria reveals anticodon-sparing strategies and domain-specific features Rna 8 1189-1232

Marshall R A, Aitken C E and Puglisi J D (2009) GTP hydrolysis by IF2 guides progression of the ribosome into elongation Molecular cell 35 37-47

Masuda T, Petrov A N, lizuka R, Funatsu T, Puglisi J D, et al. (2012) Initiation factor 2, tRNA, and 50S subunits cooperatively stabilize mRNAs on the ribosome during initiation Proceedings of the National Academy of Sciences of the United States of America 109 4881-4885

Nagase T, Ishii S and Imamoto F (1988) Differential transcriptional control of the two tRNA(fMet) genes of Escherichia coli K-12 Gene 67 49-57

Nilsson A I, Zorzet A, Kanth A, Dahlstrom S, Berg O G, et al. (2006) Reducing the fitness cost of antibiotic resistance by amplification of initiator tRNA genes Proc Natl Acad Sci U S A 103 6976-6981

Nissen P, Kjeldgaard M, Thirup S, Polekhina G, Reshetnikova L, et al. (1995) Crystal structure of the ternary complex of Phe-tRNAPhe, EF-Tu, and a GTP analog Science 270 1464-1472

O'Connor M, Gregory S T, Rajbhandary U L and Dahlberg A E (2001) Altered discrimination of start codons and initiator tRNAs by mutant initiation factor 3 Rna 7 969-978

Petrelli D, Garofalo C, Lammi M, Spurio R, Pon C L, et al. (2003) Mapping the active sites of bacterial translation initiation factor IF3 Journal of molecular biology 331541 556

Petrelli D, LaTeana A, Garofalo C, Spurio R, Pon C L, et al. (2001) Translation initiation factor IF3: two domains, five functions, one mechanism? The EMBO journal 20 45604569

Qin D, Abdi N M and Fredrick K (2007) Characterization of 16S rRNA mutations that decrease the fidelity of translation initiation Rna 13 2348-2355

Raj Bhandary U L (1994) Initiator transfer RNAs J Bacteriol 176 547-552

Samhita L, Nanjundiah V and Varshney U (2014) How many initiator tRNA genes does Escherichia coli need? J Bacteriol 196 2607-2615

Samhita L, Shetty S and Varshney U (2012) Unconventional initiator tRNAs sustain Escherichia coli Proc Natl Acad Sci U S A 109 13058-13063

Schmeing T M and Ramakrishnan V (2009) What recent ribosome structures have revealed about the mechanism of translation Nature 461 1234-1242

Selmer M, Dunham C M, Murphy F V t, Weixlbaumer A, Petry S, et al. (2006) Structure of the 70S ribosome complexed with mRNA and tRNA Science 313 1935-1942

Seong B L and RajBhandary U L (1987a) Escherichia coli formylmethionine tRNA: mutations in GGGCCC sequence conserved in anticodon stem of initiator tRNAs affect initiation of protein synthesis and conformation of anticodon loop Proceedings of the National Academy of Sciences of the United States of America 84 334-338

Seong B L and RajBhandary U L (1987b) Mutants of Escherichia coli formylmethionine tRNA: a single base change enables initiator tRNA to act as an elongator in vitro Proceedings of the National Academy of Sciences of the United States of America 84 8859-8863

Shetty S, Nadimpalli H, Shah R A, Arora S, Das G, et al. (2014) An extended Shine-Dalgarno sequence in mRNA functionally bypasses a vital defect in initiator tRNA Proc Natl Acad Sci U S A 111 E4224-4233

Shetty S, Shah R A, Chembazhi U V, Sah S and Varshney U 
(2017) Two highly conserved features of bacterial initiator tRNAs license them to pass through distinct checkpoints in translation initiation Nucleic Acids Res 45 2040-2050

Shetty S and Varshney U (2016) An evolutionarily conserved element in initiator tRNAs prompts ultimate steps in ribosome maturation Proc Natl Acad Sci U S A 113 E6126E6134

Sundari R M, Stringer E A, Schulman L H and Maitra U (1976) Interaction of bacterial initiation factor 2 with initiator tRNA The Journal of biological chemistry 251 3338-3345

Tsai A, Petrov A, Marshall R A, Korlach J, Uemura S, et al. (2012) Heterogeneous pathways and timing of factor departure during translation initiation Nature 487 390393

Varshney U, Lee C P and RajBhandary U L (1993) From elongator tRNA to initiator tRNA Proc Natl Acad Sci U S A 90 2305-2309

Varshney U, Lee C P, Seong B L and RajBhandary U L (1991) Mutants of initiator tRNA that function both as initiators and elongators The Journal of biological chemistry 266 18018-18024

Varshney U and RajBhandary U L (1992) Role of methionine and formylation of initiator tRNA in initiation of protein synthesis in Escherichia coli J Bacteriol 174 7819-7826

von Pawel-Rammingen U, Astrom S and Bystrom A S (1992) Mutational analysis of conserved positions potentially important for initiator tRNA function in Saccharomyces cerevisiae Molecular and cellular biology 12 1432-1442

Winther K S and Gerdes K (2011) Enteric virulence associated protein VapC inhibits translation by cleavage of initiator tRNA Proceedings of the National Academy of Sciences of the United States of America 108 7403-7407

Wu X Q and RajBhandary U L (1997) Effect of the amino acid attached to Escherichia coli initiator tRNA on its affinity for the initiation factor IF2 and on the IF2 dependence of its binding to the ribosome The Journal of biological chemistry 272 1891-1895. 